\title{
Removal or component reversal of local geomagnetic field affects foraging orientation preference in migratory insect brown planthopper Nilaparvata lugens
}

\author{
Yingchao Zhang ${ }^{1,2}$, Weidong Pan ${ }^{\text {Corresp. 1, } 2}$ \\ ${ }^{1}$ Beijing Key Laboratory of Bioelectromagnetics, Institute of Electrical Engineering, Chinese Academy of Sciences, Beijing, China \\ 2 University of Chinese Academy of Sciences, Beijing, China \\ Corresponding Author: Weidong Pan \\ Email address: panwd@mail.iee.ac.cn
}

Background. Migratory brown planthopper Nilaparvata lugens (N. lugens) annually migrates to Northeast Asia in spring and returns to Southeast Asia in autumn. However, mechanisms for orientation and navigation during their flight remain largely unknown. The geomagnetic field (GMF) is an important source of directional information for animals (including $N$. lugens), yet the magnetic compass involved has not been fully identified.

Methods. Here we assessed the influences of GMF on the foraging orientation preference of $N$. lugens by removing or component reversal of local GMF. At the same time, we examined the role of iron-sulfur cluster assembly1 (IsCA1), a putative component of magnetoreceptor, in the foraging orientation preference of $N$. lugens under the controlled magnetic fields by RNA silencing (RNAi).

Results. We found that the near-zero magnetic field (NZMF) or vertical reversal of GMF could lead to $N$. lugens losing the foraging orientation preference, suggesting that a normal level of GMF, in the way of either intensity or inclination, was essential for the foraging orientation of $N$. lugens. Moreover, the gene knockdown of IscA1, also affected the foraging orientation preference of $N$. lugens, pointing out a potential role of IscA1 in the insects' sensing of variation in the GMF.

Discussion. These results suggested a foraging orientation preference is associated with the GMF and revealed new insights into the relationship between the IscAl and magnetosensitivity mechanism in $N$. lugens. 
1 Removal or component reversal of local geomagnetic field affects foraging orientation

2 preference in migratory insect brown planthopper Nilaparvata lugens

3 Yingchao Zhang ${ }^{1,2}$, Weidong Pan ${ }^{1,2}$

4

$5 \quad{ }^{1}$ Beijing Key Laboratory of Bioelectromagnetics, Institute of Electrical Engineering, Chinese

6 Academy of Sciences, Beijing 100190, China; ${ }^{2}$ University of Chinese Academy of Sciences,

7 Beijing 100049, China

8 Correspondence: Weidong Pan, Beijing Key Laboratory of Bioelectromagnetics, Institute of

9 Electrical Engineering, Chinese Academy of Sciences, Beijing 100190, China. E-mail:

10 panwd@mail.iee.ac.cn 


\section{Abstract}

Background. Migratory brown planthopper Nilaparvata lugens (N. lugens) annually migrates to Northeast Asia in spring and returns to Southeast Asia in autumn. However, mechanisms for orientation and navigation during their flight remain largely unknown. The geomagnetic field (GMF) is an important source of directional information for animals (including $N$. lugens), yet the magnetic compass involved has not been fully identified.

Methods. Here we assessed the influences of GMF on the foraging orientation preference of $N$. lugens by removing or component reversal of local GMF. At the same time, we examined the role of iron-sulfur cluster assembly1 (IscA1), a putative component of magnetoreceptor, in the foraging orientation preference of $N$. lugens under the controlled magnetic fields by RNA silencing (RNAi).

Results. We found that the near-zero magnetic field (NZMF) or vertical reversal of GMF could lead to $N$. lugens losing the foraging orientation preference, suggesting that a normal level of GMF, in the way of either intensity or inclination, was essential for the foraging orientation of $N$. lugens. Moreover, the gene knockdown of IscA1, also affected the foraging orientation preference of $N$. lugens, pointing out a potential role of IscA1 in the insects' sensing of variation in the GMF.

Discussion. These results suggested a foraging orientation preference is associated with the GMF and revealed new insights into the relationship between the IscA1 and magnetosensitivity mechanism in N. lugens.

Subjects: Agricultural Science, Entomology

Key words: Nilaparvata lugens; Foraging orientation; Iron-sulfur cluster assembly1; Magnetosensitivity 


\section{Introduction}

The brown planthoppers, Nilaparvata lugens (N. lugens), are recognized as a major migratory rice pest and virus vector. Adults exhibit wing dimorphism with macropterous and brachypterous phenotypes. The macropterous insects have functional wings for long-distance migration and the brachypterous individuals are non-migratory (Guerra 2011). In East Asia, N. lugens adults overwinter in Vietnam and southern China. In order to find enough food and suitable living environment, they migrate to Northeast Asia in spring and return back to Southeast Asia in autumn (Kisimoto 1976; Cheng et al. 1979). N. lugens is a nocturnal species that usually takes flight sometime between sunset and sunrise (Kisimoto 1979). However, the mechanisms for orientation and navigation during their flight remain largely unknown.

Many insects utilize magnetic information as a compass for their orientation and navigation. For instance, the migratory butterflies such as the sulphur butterflies Aphrissa statira or the monarch butterfly Danaus plexippus can orient with a sun compass, but are also observed migrating directionally under overcast skies. Accordingly, it has been confirmed that a magnetic compass was involved in both species (Srygley et al. 2006; Guerra et al, 2014). It is also the case for some migratory moths as the Mythimna separata that maintain migratory direction in the night sky (Xu et al., 2017). The use of magnetic compass has also been found in foraging of insects, including honeybee and ants. For honeybees, altering the GMF causes misdirection in the waggle dance, which is performed in the foraging trip, while there is no misdirection when dance orient along magnetic field lines (Towne \& Gould, 1985). Ant foragers can be trained to recognize the location of a food source in the magnetic field and their orientation will turn according to the artificial magnetic fields (Anderson \& Meer, 1993; Camlitepe \& Stradling, 1995). In this respect, the use of magnetic compass as a key mechanism involved in insect directional movements appears feasible. This perspective was further supported by the findings of magnetic particles in insect tissues (Gould et al. 1978; Wajnberg, 1999; Chambarelli et al. 2008; Pan et al. 2016), which could become the substrate for the magnetic compass. However, until now no behavioral observations 
63 have been confirmed the presence of magnetic compass in N. lugens.

64 So far there are two models which are most popular to explain how animals detect the 65 magnetic field: the magnetite-based mechanisms (Beason 1986; Kirschvink \& Gould 1981; 66 Lohmann 2010) and the radical pair-based mechanisms (Ritz et al. 2000). Recently, a light67 magnetism-coupled magnetosensitivity model has been proposed, in which the homolog of the bacterial iron-sulfur cluster assembly, IscA1, that forms a complex with cryptochromes is suggested to serve as a putative magnetic protein biocompass (Qin et al. 2016). The iron-sulfur cluster proteins are ancient macromolecules with highly conserved structures. They have many functions including iron homeostasis, electron transfer, metabolic catalysis, nitrogen fixation, regulation of gene expression and the detection of reactive oxygen species (Beinert et al. 1997; Beinert 2000). Qin et al. (2016) reported that the protein complex exhibited strong intrinsic magnetic polarity and rotated in synchrony with the external magnetic field. Previously we have found the IscA1 gene in $N$. lugens showed up-regulated mRNA expression during the period of migration (Xu et al. 2017). For the macropterous migratory N. lugens, compared with the GMF, the mRNA expression of the IscA1 gene and the cryptochrome1 gene were up-regulated under the magnetic fields of 0.5 millitesla $(\mathrm{mT})$ and $1 \mathrm{mT}$ in strength. The findings revealed that the expression of IscA1 and cryptochromes in $N$. lugens exhibited coordinated responses to the magnetic field, suggesting the potential associations between IscA1 and the magnetic sensory system.

In this study, we demonstrated the effects of altered GMF, i.e., near-zero magnetic field (NZMF) or components reversal of GMF, on the foraging orientation in N. lugens. By using the RNA silencing (RNAi) on $N$. lugens, the functional role of IscA1 was investigated.

\section{Materials and methods}

\subsection{Insect stock}

Experiments were performed at Beijing Key Laboratory of Bioelectromagnetics, Institute of 
Electrical Engineering, Chinese Academy of Sciences, Beijing, China. The insects were reared in climate chambers at day/night temperatures of $(27 \pm 1)^{\circ} \mathrm{C} /(26 \pm 1)^{\circ} \mathrm{C}$ on susceptible Taichuang Native 1 (TN1) rice plant under 14:10 h light: dark cycle and 70 $\pm 5 \%$ humidity (Wan et al. 2015) and the environmental conditions of the chambers was the same in the entire experiment. The TN1 rice plants were prepared in advance, and used as the food for the insects when they grew up to 10 $\mathrm{cm}$ height. The migratory macropterous female and male adults were selected from the same generation for the successive generations (Wan et al. 2016).

\subsection{Magnetic field devices setup}

The GMF used in the experiment (total intensity: $52487 \pm 841 \mathrm{nT}$; declination $5.30 \pm 0.59^{\circ}$; inclination $\left.56.29 \pm 1.02^{\circ}\right)$ were the local GMF at $\left(39^{\circ} 59^{\prime} 14^{\prime \prime} \mathrm{N}, 116^{\circ} 19^{\prime} 21^{\prime \prime} \mathrm{E}\right)$. The artificial magnetic fields were produced using a Helmholtz coil system (Fig. 1). For NZMF, the Helmholtz coils were used to produce a near-zero magnetic field region with an average intensity of $\sim 500 \mathrm{nT}$ at a center spherical space $(150 \mathrm{~mm}$ in radius). For component reversal of GMF, the Helmholtz coils were used to generate a magnetic field with twice intensity and reversed direction to offset either the horizontal component or the vertical component of GMF, producing a reversed inclination with the same intensity but reversed component of GMF. Routinely before and after each experiment, we measured the three components of GMF using a fluxgate magnetometer (Model 191A, Honor Top Magnetoelectric Technology Co. Ltd., Qingdao, China; sensitivity: $\pm 1 \mathrm{nT})$ to modulate the electric current of the coil pairs to produce the required intensity for NZMF.

\subsection{Cross-tube choice system and foraging orientation experiments}

The choice system consisted of a cross tube which was embedded in a plastic square. The length of each arm of the cross tube was $110 \mathrm{~mm}$. The width was $20 \mathrm{~mm}$ and the depth was $30 \mathrm{~mm}$. The cross tube was covered with a plastic lid of same size. There were small holes at each arm end for air flowing through. A lamp (15W, $\lambda=320-680 \mathrm{~nm})$ was installed as the light source (there is faint light when the $N$. lugens takes off at the sunset or sunrise) with 400 lumen of lumination 
113 intensity at the cross tube. The coil system was covered by a shade cloth during the experiment to 114 shield the external environment (Fig. 1). During the experiment, the cross tube was placed

115 horizontally inside the Helmholtz coils and the arms of the cross tube were oriented towards four 116 cardinal points. The cardinal points used in the experiments were the same. Two cross tubes were 117 used in the experiment, one containing food as a reward and the other without food. Ten fresh rice 118 seedlings of susceptible variety of TN1 (Fu et al. 2001) was placed at one arm end as food reward.

The experiment was conducted in two parts: the first part of the trial was to provide food as a stimulus that the insects might associate with magnetic cues under the normal GMF, and the second part of the trial was to test for a disruption of their ability to exhibit this learned directional preference when the GMF was altered. Adult insects within $48 \mathrm{~h}$ of emergence regardless of gender or mating status were collected from the rearing colony and introduced into the center of the cross tube using a self-made insect suction-implement. The rice seedings were placed $70 \mathrm{~mm}$ away from the center (Fig. 2A). For the first part of the trial, the insects gathering around the rice seedlings (40 \pm 13 insects) were eventually collected (Fig. 2B) using the same suction-implement into a vial and afterwards a new cross tube with no rice seedlings inside was placed horizontally in the Helmholtz coils. For the second part of the trial, the collected insects in the vial were replaced in the center of the new cross tube using the suction-implement (Fig. 2C) and the insects moving to each of the four arms of the new cross tube were recorded for $0.5 \mathrm{~h}$ (Fig. 2D). The entire experiment was performed at room temperature $\left(26 \pm 1^{\circ} \mathrm{C}\right)$ and each magnetic field setup was performed individually for 12 replicates and the total number of insects tested was $511 \pm 69$.

\subsection{The effects of IscA1 gene silencing on the orientation of N.lugens}

The IscA1 gene was previously cloned in Nilaparvata lugens and the results showed that the gene expression reached the peak at the third day after emergence (Xu et al. 2017), so adults of $1^{\text {st }}$ day after emergence were selected for RNAi according to Liu et al (2010). The primers (Table 1) were designed based on the fragment sequence that was searched from transcriptome of $N$. lugens 
138 by local BLAST search. The dsRNA of IscA1 gene was designed at two different regions: nearing 139 the 3 'end (dsRNA1) and nearing the 5'end (dsRNA2). Insects were anesthetized with $\mathrm{CO}_{2}$ for 30s 140 at $\mathrm{PCO}_{2}=1 \mathrm{mPa}$ and immobilized on a $1.5 \%$ agarose plate with abdomen upward. Each insect was 141 injected with 250ng (50nl in volume) dsRNA. On the second day after injection (24-48h), the 142 injected insects were collected and placed inside the GMF for foraging orientation test with the 143 cross-tube system. The cross-tube behavioral trials for the RNAi insects were conducted in the 144 same manner as described in section 2.3 as part of a two-step trial. A total number of 700 insects were used for the experiments. To ensure the silencing efficiency, the expression level of IscAI gene was investigated before and after the behavioral test using 3 pools of 6 insects for each group by fluorescence-based quantitative real-time PCR (q-PCR) (Bustin et al. 2009). The whole body of adult $N$. lugens was used for sampling and all the samples were collected during the same time period (19:00-20:00 hours). Total mRNA was extracted by TRIzol reagent (Invitrogen, USA). The quality of samples was determined by spectrophotometric optical density (OD) 260/280 and 2\% agarose gel electrophoresis. The cDNA templates were synthesized with $1 \mu \mathrm{g}$ of total RNA using PrimeScript $^{\mathrm{TM}}$ RT reagent Kit with gDNA Eraser (TaKaRa, Tokyo, Japan). Each cDNA product was diluted with sterilized double- distilled water. The house-keeping gene for the q-PCR was actin1 (GenBank accession No. EU179846, and the PCR amplification efficiency was established by means of calibration curves (Bustin et al., 2009). The optimized thermal program was designed according to the kit instructions. Quantification of the transcript level of genes was conducted according to the ${ }^{{ }^{\Delta}} \mathrm{Cq}$ method (Livak and Schmittgen, 2001). RNA samples were analyzed independently for three times. The dsRNA of green fluorescent protein-GFP (GenBank accession No. U76561) was injected into the $N$. lugens as the control.

\subsection{Statistical analysis}

All data were analyzed using SPSS 20.0 (IBM Inc., Armonk, U.S.A.). The chi-square test was used to analyze the ratio of the distribution of insects in four directions. If there was significant difference, Bonferroni correction was used to analyzed the difference between every two 
164 directions. One-way ANOVA was used to analyze the gene expression. Significant differences

165 between $d s G F P$ (control) and $d s N l-I s c A 1$ injection treatments were determined by one-way 166 ANOVA at $p<0.05$

167

168

169

170

\section{Results}

\subsection{The foraging orientation preference of N.lugens in the GMF vs NZMF}

In the GMF, $N$. lugens showed the highest preference of foraging orienting to the north direction with original food $\left(\chi^{2}=108.48, p<0.001\right)$. The percentage of individuals orienting to the north was $39.06 \%$, which was significantly higher than that to south $14 \%\left(\chi^{2}=98.481, p<0.001\right)$, west $25.40 \%\left(\chi^{2}=26.169, p<0.001\right)$ and east $21.39 \%\left(\chi^{2}=45.652, p<0.001\right.$, Fig. $\left.3 \mathrm{~A}\right)$. In the NZMF, however, $N$. lugens were relatively equally distributed and the percentage of individuals orienting to the north, south, west and east direction was $25.46 \%, 22.22 \%, 27.55 \%$ and $24.77 \%$, respectively $\left(\chi^{2}=10.261, p=0.088\right)($ Fig. 3B).

\subsection{The foraging orientation preference of N.lugens in the horizontal or vertical component} reversal of GMF

In order to study how the GMF affects the foraging orientation ability of $N$. lugens, we conducted behavior experiment in the horizontal or vertical component reversal of GMF. In the horizontal component reversal of GMF, most of $N$. lugens were distributed in the north $\left(\chi^{2}=87.872\right.$, $p<0.001$, Fig. 4A), similar to that in GMF. In the vertical component reversal of GMF, the percentage of insects orienting to the north, south, west and east direction observed as $25.18 \%$, $21.74 \%, 29.53 \%$ and $23.55 \%$, respectively $\left(\chi^{2}=9.371, p=0.102\right)($ Fig. $4 \mathrm{~B})$.

\subsection{The IscA1 gene knockdown affected the foraging orientation preference of N.lugens}

The q-PCR results showed that the mRNA expression of IscAl was effectively downregulated after the gene silencing. Both of the silencing efficiencies of dsRNA1 and dsRNA2 were over $80 \%$ within $24 \mathrm{~h}$ (before the behavioral experiment) and $48 \mathrm{~h}$ (after the behavioral 
188

189

190

191

192

193

194

195

196

197

198

199

200

201

202

203

204

205

206

207

208

209

210

211

212

experiment) after microinjection (Fig. 5). Since the $N$. lugens preferred the north foraging direction in GMF or horizontal component reversal of GMF, we chose these conditions to investigate whether IscA1 gene silencing would affect the insects' choice of direction. In these two magnetic fields, most of the $N$. lugens with IscA1 gene silencing distributed in the west, followed by the north, south and east (Fig. 6). Compared with the wild type, the percentage of IscA1 gene knockdown $N$. lugens distributed in the north direction revealed significantly decreased from $39.06 \%$ to $28.82 \%$ in GMF $\left(\chi^{2}=13.183, p<0.001\right.$, Fig. 6 A $)$ and to $28.57 \%$ in the horizontal component reversal of GMF $\left(\chi^{2}=10.151, p<0.001\right.$, Fig. 6B $)$.

\section{Discussion}

Previous studies revealed that exposure of both small brown planthopper and brown planthopper to NZMF delayed egg and nymphal developmental durations and decreased adult weight and female fecundity of insects (Wan et al. 2014). In addition to growth and development, the NZMF also affected positive phototaxis and flight capacity of the white-backed planthopper Sogatella furcifera (Wan et al. 2016). Exposure to enhanced GMF also reduced the phototaxis of $N$. lugens (Zhang et al. 2019). In this study, we found majority of the insect $N$. lugens initially tested preferred north foraging orientation in the GMF, which was consistent with the field observation that $N$. lugens migrate to Northeast Asia under spring/summer-like conditions (Kisimoto 1976; Cheng et al. 1979). In our experiment, the first part of the trial with rice seedlings to the north is to provide the opportunity for the insects to associate magnetic field information under the normal GMF with the presence of food in a particular direction, and the second part was designed to test whether the changed GMF (either NZMF in Figure 3 or component reversal in Figure 4) affected their ability to exhibit this learned directional foraging preference. As $S$. furcifera and $N$. lugens are both migratory insect pests of rice crops, the reported effects of removal of GMF suggest a role of the GMF, in terms of energy regulation or flight orientation, in their local scale foraging movement and also possibly their long-distance migration. 
Generally, the inclination compass worked when the vertical component of the geomagnetic

214

215

216

217

218

219

220

221

222

223

224

225

226

227

228

229

230

231

232

233

234

235

236

237

238 field was reversed, as it was shown that the mealworm beetle Tenebrio molitor significantly turned their preferred direction by $180^{\circ}$ when the vertical component was reversed (Vácha et al., 2008). It has also been reported that birds could not distinguish between north and south by the polarity of the geomagnetic field, but could distinguish poleward and equatorward movement by the inclination of the field lines (Wiltschko and Wiltschko, 1996). In this study, when the vertical component of GMF was reversed, $N$. lugens individuals showed no significant foraging orientation. Thus, the foraging orientation of $N$. lugens in the vertical component reversed magnetic field was partially consistent to the inclination compass observed in monarch butterfly (Guerra et al. 2014). In this study, when the vertical component was reversed, N.lugens didn't distribute in the opposite direction as did the monarch butterfly. We speculate that this may be due to the existence of multiple compasses involved in insect orientation. N.lugens migrates in the sunset or sunrise (Kisimoto 1979), so it's likely that a light-based mechanism of magnetoreception is also involved. Here our results suggested that a magnetic compass aided the foraging orientation preference of the migratory insect $N$. lugens, but $N$. lugens might also use other orientation cues.

As the homologue of bacterial iron-sulfur cluster assembly, the IscA1 has been found in most prokaryotic and eukaryotic organisms with highly conserved structures. The inhibition of IscA1 could disrupt circadian rhythms in the fruit fly (Mandilaras \& Missirlis 2012). Moreover, it was found that knockdown of the IscA1 led to anemia in zebra fish (Nilsson et al. 2009). Currently a protein complex formed by the IscA1 interacting with cryptochromes was proposed as a putative magnetoreceptor and the protein crystal was claimed to exhibit strong magnetic polarity in response to an external magnetic field (Qin et al. 2016). The findings, however, have raised considerable controversy due to the broad interpretation of its biological meaning as well as the limitation of in vitro experiments (Friis et al. 2017; Hochstoeger et al. 2016; Pang et al. 2017). Therefore, an independent investigation should be performed to clarify as far as possible whether the IscA1 is involved in specific processes of magnetosensitivity in terms of functional behaviors

Peer) reviewing PDF | (2020:08:52068:2:0:NEW 21 Sep 2021) 
239 as navigation and orientation in long-distance migration of animals (Meister 2016; Nicholls 2016).

240 In this study, our results showed that the foraging orientation preference of insects was affected by

241 the IscA1 gene knockdown under varying GMF, providing direct evidence of IscA1 involved in

242 magnetosensitivity of $N$. lugens. Meanwhile, biogenic magnetic particles were proposed to

243 function as a hypothetic magnetoreceptor: the external magnetic field can affect the internal

244 magnetite clusters leading to magnetic orientation loss (Davila et al. 2005). Previously we have

245 detected magnetic particles in N. lugens (Pan et al. 2016) which also provides additional support

246 for a magnetic sense in $N$. lugens. Whether the IscA1 protein is functionally linked to formation

247 of magnetic particles and how these hypothetic magnetoreceptors work in synergism in vivo

248 remains to be further elucidated.

\section{Conclusion}

This study provided behavioral evidence that the foraging orientation preference of the migratory insect $N$. lugens is affected by removal or component reversal of local GMF. When the vertical component of GMF was reversed, the insects showed no significant foraging orientation preference, suggesting the potential use of inclination compass-aided orientation in $N$. lugens. The foraging orientation preference of $N$. lugens was also affected by the IscA1 gene knockdown, providing a feasible mechanistic explanation for the insects' sensing of variation in the GMF. Further work is needed to investigate the potential associations between the IscA1 and magnetic particles in terms of the magnetosensitivity mechanism in N. lugens.

\section{Acknowledgments}

We thank Prof. Hongxia Hua of College of Plant Science and Technology of Huazhong Agricultural University providing the original laboratory stock of the $N$. lugens. We also thank Space Electromagnetic Environment Laboratory for providing geomagnetic data.

\section{Funding}


264 31870367, 31470454, 31672019, 31701787), the National Department of Public Benefit Research 265 Foundation (201403031), the Natural Science Foundation of Jiangsu Province Youth Fund 266 (SBK2016043525) and the China Postdoctoral Science Foundation (2016M590470). The funders

267 had no role in study design, data collection and analysis, decision to publish, or preparation of the 268 manuscript.

\section{Grant Disclosures}

270 The following grant information was disclosed by the authors:

271 National Natural Science Foundation of China (NSFC): 31670855, 31870367, 31470454, $27231672019,31701787$.

273 National Department of Public Benefit Research Foundation: 201403031.

274 Natural Science Foundation of Jiangsu Province Youth Fund: SBK2016043525.

275 China Postdoctoral Science Foundation: 2016M590470.

\section{Competing Interests}

277 The authors declare that they have no competing interests.

\section{Author Contributions}

279

- Yingchao Zhang conceived and designed the experiments, performed the experiments, analyzed the data, prepared figures and/or tables, authored or reviewed drafts of the paper, and approved the final draft.

- Weidong Pan conceived and designed the experiments, approved the final draft.

\section{Data availability}

The following information was supplied regarding data availability:

The raw data are available in a Supplemental File. 
286

287

288

289

290

291

292

293

294

295

296

297

298

299

300

301

302

303

304

305

306

307

308

309

310

311

\section{References}

Anderson JB, Meer RKV. 1993. Magnetic orientation in the fire ant, Solenopsis invicta. Naturwissenschaften, 80:568-570. DOI:10.1007/BF01149274.

Beason RC. 1986. Magnetite biomineralization and magnetoreception in organisms: a new biomagnetism. by Joseph L. Kirschvink; Douglas S. Jones; Bruce J. MacFadden. Quarterly Review of Biology, 61, 429-430. DOI:10.1016/0300-9629(87)90453-1.

Beinert H. 2000. Iron-sulfur proteins: ancient structures, still full of surprises. Journal of Biological Inorganic Chemistry, 5, 2-15. DOI:10.1007/s007750050002.

Beinert H, Holm RH, Münck E. 1997. Iron-sulfur clusters: nature's modular, multipurpose structures. Science, 277, 653-659. DOI:10.1126/science.277.5326.653.

Bustin SA, Benes V, Garson JA, Hellemans J, Huggett J, Kubista M, Mueller R, Nolan T, Pfaffl MW, Shipley GL, Vandersompele J, Wittwer C. 2009. The MIQE guidelines: minimum information for publication of quantitative real-time PCR experiments. Clinical Chemistry, 55, 611-622. DOI: 10.1373/clinchem.2008.112797.

Camlitepe Y,Stradling DJ. 1995. Wood Ants Orient to Magnetic Fields. Proceedings of the Royal Society B: Biological Sciences.261,37-41. DOI:10.2307/50044.

Chambarelli LL, Pinho MA, Abraçado LG, Esquivel DMS, Wajnberg E. 2008. Temporal and preparation effects in the magnetic nanoparticles of apis mellifera, body parts. Journal of Magnetism \& Magnetic Materials, 320, 207-210. DOI:10.1016/j.jmmm.2008.02.049.

Cheng SN, Chen JC, Si H, Yang LM, Chu TL, Wu CT, Chen JK, Yang CS. 1979. Studies on the migrations of brown planthopper Nilaparvata lugens Stal.Acta Entomologica Sinica, 22(1), $1-21$.

Davila AF, Winklhofer M, Shcherbakov VP, Petersen N. 2005. Magnetic pulse affects a putative magnetoreceptor mechanism. Biophysical Journal, 89, 56-63. DOI: 10.1529/biophysj.104.049346.

Fu Q, Zhang ZT, Hu C, Lai FX, Sun ZX. 2001. A chemically defined diet enables continuous 
312

313

314

315

316

317

318

319

320

321

322

323

324

325

326

327

328

329

330

331

332

333

334

335

336

337

rearing of the brownplanthopper, Nilaparvata lugens (stal) (homoptera :

delphacidae). Applied Entomology \& Zoology, 36, 111-116. DOI:10.1016/S00078506(07)60148-6.

Friis I, Sjulstok E, Solov'Yov IA. 2017. Computational reconstruction reveals a candidate magnetic biocompass to be likely irrelevant for magnetoreception. Scientific Reports, 7, 13908. DOI:10.1038/s41598-017-13258-7.

Gould JL, Kirschvink JL, Deffeyes KS. 1978. Bees have magnetic remanence. Science, 201, 1026-1028. DOI: 10.1126/science.201.4360.1026.

Guerra PA. 2011. Evaluating the life-history trade-off between dispersal capability and reproduction in wing dimorphic insects: a meta-analysis. Biological Reviews, 86, 813-835. DOI:10.1111/j.1469-185X.2010.00172.X.

Guerra PA, Gegear RJ, Reppert SM. 2014. A magnetic compass aids monarch butterfly migration. Nature Communications. 5, 4164-4164. DOI: 10.1038/ncomms5164.

Hochstoeger T, Nimpf S, Keays D, 2016. ISCA1 and CRY4: An improbable proposition. BioRxiv, 094458. DOI:10.1101/094458.

Kirschvink JL, Gould JL. 1981. Biogenic magnetite as a basis for magnetic field detection in animals. Biosystems, 13, 181-201. DOI: 10.1016/0303-2647(81)90060-5.

Kisimoto R. 1976. Synoptic weather conditions inducing long-distance immigration of planthoppers, Sogatella furcifera Horvath and Nilaparvata lugens Stal. Ecological Entomology, 1, 95-109. DOI:10.1111/j.1365-2311.1976.tb01210.x

Kisimoto R. 1979. Brown planthopper migration. Brown Planthopper Threat to Rice Production in Asia. pp:113-124. International Rice Research Institute, Los Banos.

Lohman KJ. 2010. Animal behaviour: magnetic-field perception. Nature, 464, 1140-1142. DOI:10.1038/4641140a.

Liu SH, Ding ZP, Zhang CW, Yang BJ, Liu ZW. 2010. Gene knockdown by intro-thoracic injection of double-stranded rna in the brown planthopper, Nilaparvata lugens. Insect 
Biochemistry \& Molecular Biology, 40, 666-671. DOI:10.1016/j.ibmb.2010.06007.

339

340

341

342

343

344

345

346

347

348

349

350

351

352

353

354

355

356

357

358

359

360

361

362

363

Mandilaras K, Missirlis F. 2012. Genes for iron metabolism influence circadian rhythms in Drosophila melanogaster. Metallomics, 4, 928-936. DOI:10.1039/c2mt20065a.

Meister M. 2016. Physical limits to magnetogenetics. Elife, 5, e17210. DOI:10.7554/eLife.17210.

Nilsson R, Schultz IJ, Pierce EL. 2009. Discovery of genes essential for heme biosynthesis through large-scale gene expression analysis. Cell Metabolism, 10, 119-130. DOI:10.1016/j.cmet.2009.06.012.

Pan WD, Wan GJ, Xu JJ, Li XM, Liu YX, Qi LP, Chen FJ. 2016. Evidence for the presence of biogenic magnetic particles in the nocturnal migratory brown planthopper, Nilaparvata lugens. Scientific Reports, 6, 18771. DOI:10.1038/srep18771.

Pang K, You H, Chen Y, Chu P, Hu M, Shen J, Guo W, Xie C, Lu B. 2017. MagR alone is insufficient to confer cellular calcium responses to magnetic stimulation. Frontiers in Neural Circuits, 11, 11. DOI:10.3389/fncir.2017.00011.

Qin SY, Yin H, Yang CL, Dou YF, Liu ZM, Zhang P, Yu H, Huang YL, Feng J, Hao J, Deng LZ, Yan XY, Dong XL, Zhao ZX, Jiang TJ, Wang HW. Luo SJ, Xie C. 2016. A magnetic protein biocompass. Nature Materials, 15, 217-226. DOI:10.1038/nmat4484.

Ritz T, Adem S, Schulten K. 2000. A model for photoreceptor-based magnetoreception in birds. Biophysical Journal, 78, 707-718. DOI:10.1016/S0006-3495(00)76629-X.

Srygley RB, Dudley R, Oliveira EG, Riveros AJ. 2006. Experimental evidence for a magnetic sense in Neotropical migrating butterflies (Lepidoptera: Pieridae). Animal behaviour, 71, 183-191. DOI:10.1016/j.anbehav.2005.04.013.

Towne WF， Gould JL. 1985. Magnetic field sensitivity in honeybees. Magnetite Biomineralization and Magnetoreception in Organisms. pp385-406. New York: Plenum. DOI: $10.1007 / 978-1-4613-0313-8-18$.

Vácha M, Drš tková D, Pů žová T. 2008. Tenebrio beetles use magnetic inclination compass. 
Naturwissenschaften, 95, 761-765. DOI: 10.1007/s00114-008-0377-9

Wajnberg E, Linhares MP. 1999. Evidence for magnetic material in the fire ant solenopsis sp. by electron paramagnetic resonance measurements. measurements. Naturwissenschafte, 86, 30-32. DOI: 10.1007/s001140050564.

Wan GJ, Jiang SL, Zhao ZC, Xu JJ, Tao XR, Sword GA, Gao YB, Pan WD, Chen FJ. 2014. Bioeffects of near-zero magnetic fields on the growth, development and reproduction of small brown planthopper, Laodelphax striatellus and brown planthopper, Nilaparvata lugens. Journal of Insect Physiology, 68, 7-15. DOI: 10.1016/j.jimsphys.2014.06.016.

Wan GJ, Wang WJ, Xu JJ, Yang QF, Dai MJ, Zhang FJ, Sword GA, Pan WD, Chen FJ. 2015. Cryptochromes and hormone signal transduction under near-Zero magnetic fields: new clues to magnetic field effects in a rice planthopper. Plos One, 10, e0132966. DIO: 10.1371/journal.pone.0132966.

Wan GJ, Yuan R, Wang WJ, Fu KY, Zhao JY, Jiang SL, Pan WD, Sword GA, Chen FJ. 2016. Reduced geomagnetic field may affect positive phototaxis and flight capacity of a migratory rice planthopper. Animal Behaviour, 121, 107-116. DOI:10.1016/j.anbehav.2016.08.024.

Wan GJ, Liu RY, Li CX, He JL, Pan WD, Sword GAm Hu G, Chen FJ. 2020. Change in geomagnetic field intensity alters migration-associated traits in a migratory insect. Biology Letters, 16 (4), DOI: 10.1098/rsbl.2019.0940

Wan GJ, Jiang SL, Zhang M, Zhao JY, Zhang YC, Pan WD, Sword GA, Chen FJ. 2021. Geomagnetic field absence reduces adult body weight of migratory insect by disrupting feeding behavior and appetite regulation. Insect Science, 28(1):251-260. DOI: 10.1111/1744-7917.12765.

Wiltschko W, Wiltschko R. 1996. Magnetic orientation in birds. Journal of Experimental Biology, 199, 29-38. DOI: 10.1007/978-1-4615-6787-5_2

Xu JJ, Pan W, Zhang YC, Li Y, Wan GJ, Chen FJ, Sword GA, Pan WD. 2017. Behavioral evidence for a magnetic sense in the oriental armyworm, Mythimna separata. Biology 
Open, DOI: 10.1242/bio.022954.

391 Xu JJ, Zhang YC, Wu JQ, Wang WH, Li Y, Wan GJ, Chen FJ, Sword GA, Pan WD. 2019.

392 Molecular characterization, spatial-temporal expression and magnetic response patterns of

393 the iron-sulfur cluster assembly1 (IscA1) in the rice planthopper, Nilaparvata lugens. Insect

$394 \quad$ Science, 26, 413-423. DOI:10.1111/1744-7917.12546.

395

Zhang YC, Wan GJ, Wang WH, Li Y, YuY, Zhang YX, Chen FJ, Pan WD. 2019. Enhancement

396 of the geomagnetic field reduces the phototaxis of rice brown planthopper Nilaparvata lugens associated with frataxin down-regulation. Insect Science, DOI: 10.1111/1744-

398 7917.12714

399 


\section{Table $\mathbf{1}$ (on next page)}

Primers used in the experiments. 


\begin{tabular}{ll}
\hline Primer & Sequence (5' to $3^{\prime}$ ) \\
\hline NL-IscA1-dsRNA1-F & TAATACGACTCACTATAGGGAGAGCAGCACTGGTTTTGAC \\
& AC \\
NL-IscA1-dsRNA1-R & TAATACGACTCACTATAGGGGCTTTTGCATCTATTATGACG \\
& A \\
NL-IscA1-dsRNA2-F & TAATACGACTCACTATAGGGGAGAAAGGAAAGTTTGACGA \\
NL-IscA1-dsRNA2-R & TAATACGACTCACTATAGGGAAAACTCCAAAGGAGAAAAT \\
NL-GFP-dsRNA-F & TAATACGACTCACTATAGGGACGTAAACGGCCACAAGTTA \\
NI-GFP-dsRNA-R & TAATACGACTCACTATAGGGTGTTCTGCTGGTAGTGGTCG \\
NL-IscA-qPCR-F & AGACTCATATAGACGATGTTAA \\
NL-IscA-qPCR-R & CCTCCAATGTCCTCTAAC \\
NL-actin-qPCR-F & CCAACCGTGAGAAGATGACC \\
NL-actin-qPCR-R & GATGTCACGCACGATTTCAC \\
&
\end{tabular}


Figure 1

The magnetic field generating device and the cross-tube choice chamber.

The Helmholtz coil system consisted of three independent coil pairs and each pair of coils was powered separately which could produce the magnetic field. The cross-tube choice chamber was placed horizontally inside the coil and a shade cloth covered the coil system during the experiment.

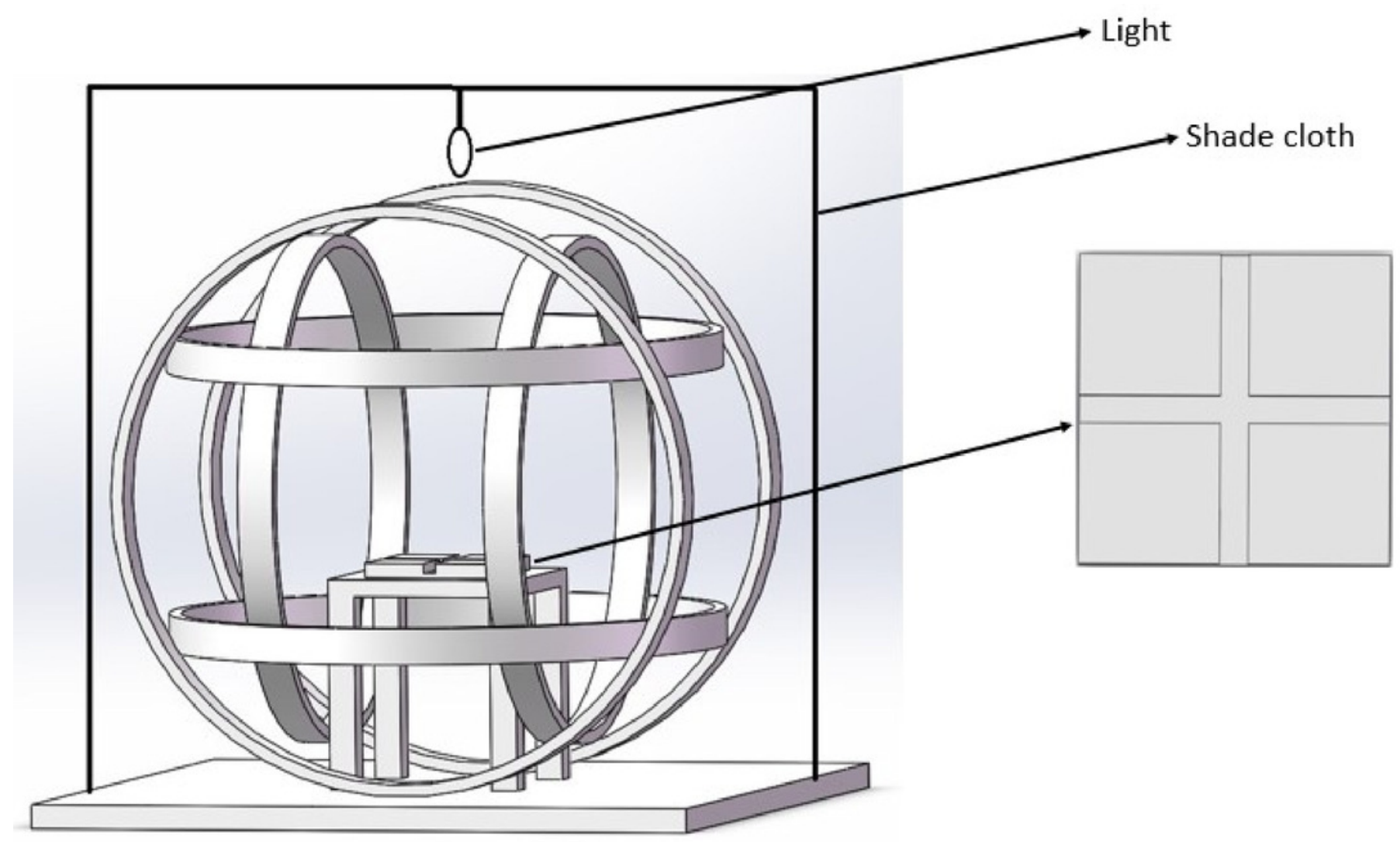


Figure 2

The flow chart of cross-tube choice experiments.

(A) The insects were put in the cross tube with food source (green lines) inside. (B) Ten hours later, the insects gathered around in the food source were collected. (C) The collected insects were put in a new cross tube without food source. (D) After half an hour, the distribution of the insects was recorded.

A

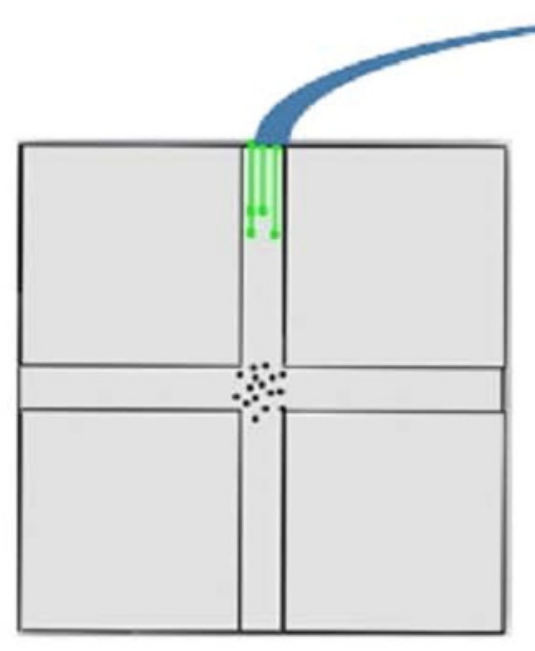

D

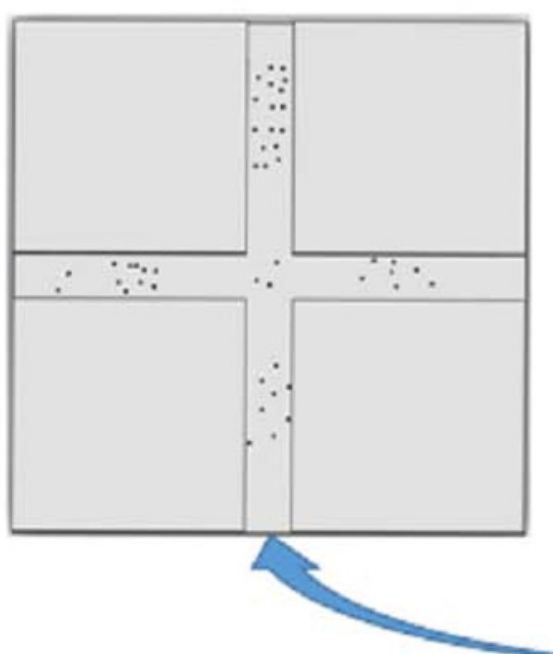

B

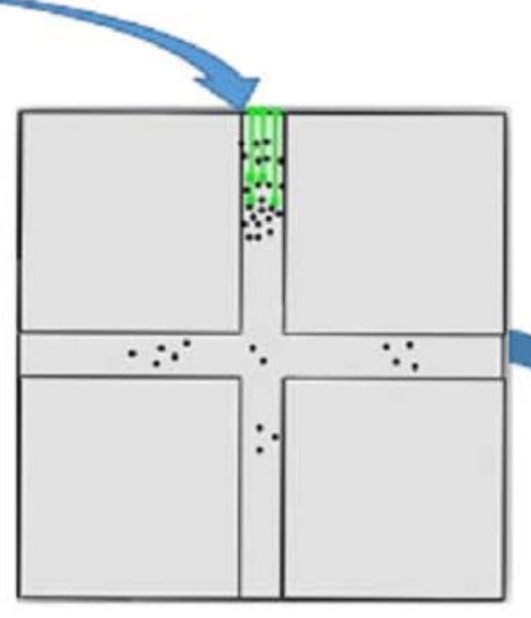

C

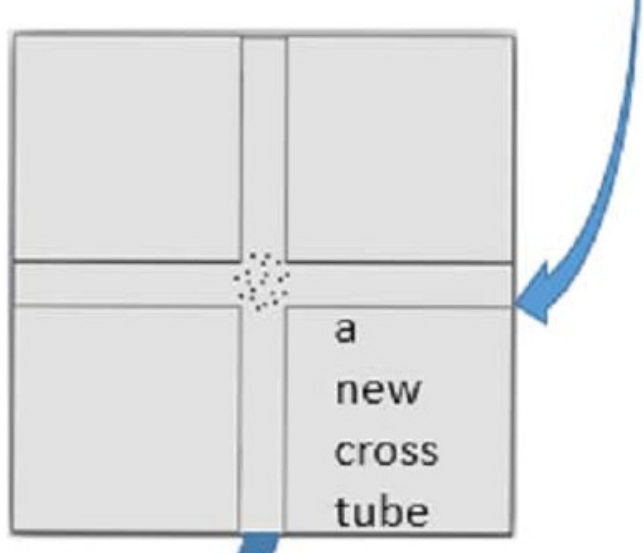




\section{Figure 3}

The distribution of N.lugens in local geomagnetic filed (GMF, A) and near-zero magnetic field (NZMF, B) with food source initially located in the north direction.

A total number of insects with $\mathrm{N}=241,87,157,132$ for the distribution of insects in GMF and $\mathrm{N}=110,96,119,107$ for the distribution of insects in NZMF were used for experiments.

Different lowercase letters indicate significant differences among directions by chi-square test at $p<0.05$.
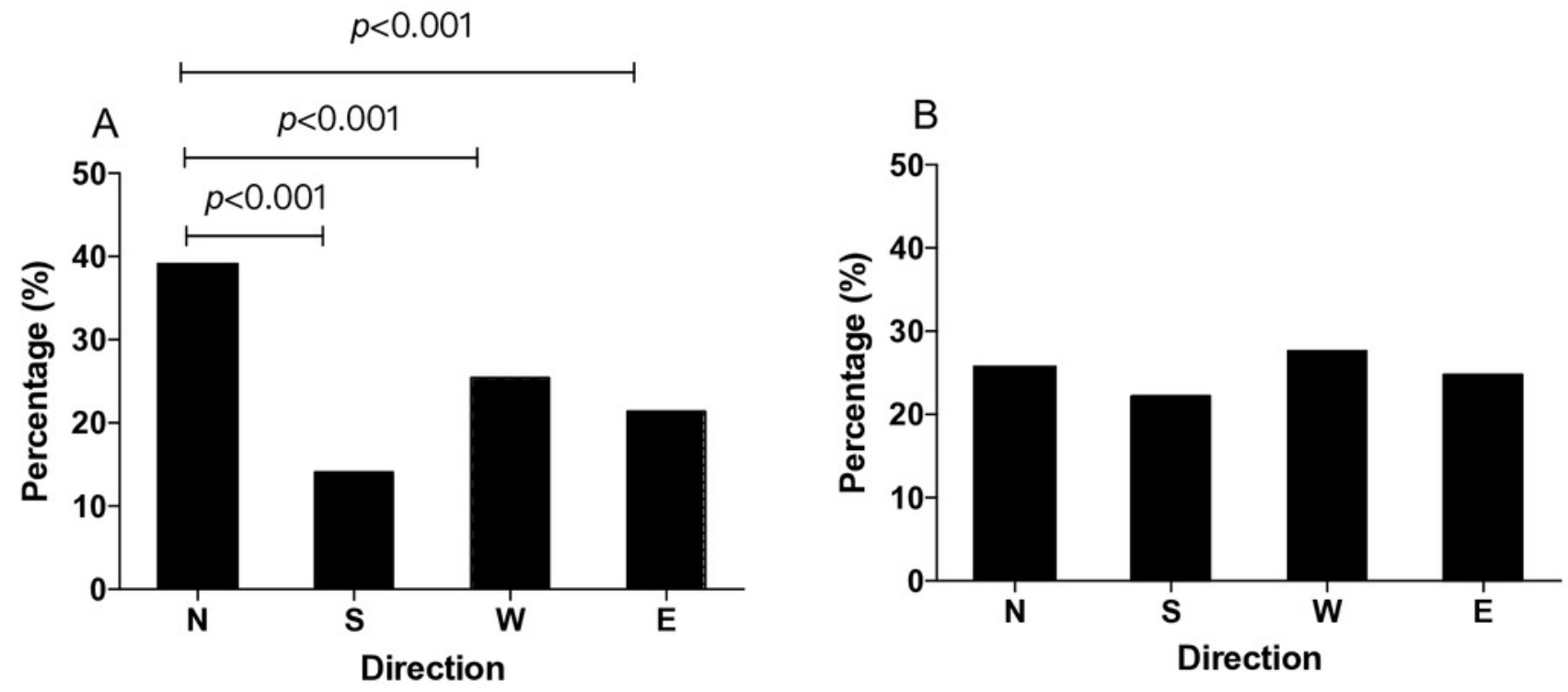


\section{Figure 4}

The distribution of $N$. lugens under horizontal or vertical component reversal of local geomagnetic field (GMF) with food source initially located in the north direction.

(A) Horizontal component reversal of GMF. (B) Vertical component reversal of GMF. A total number of insects with $\mathrm{N}=191,93,122,91$ for the distribution in horizontal component reversal of GMF and $\mathrm{N}=139,120,163,130$ for the distribution in vertical component reversal of GMF were used for experiments. Different lowercase letters indicate significant differences among directions by chi-square test at $p<0.05$.
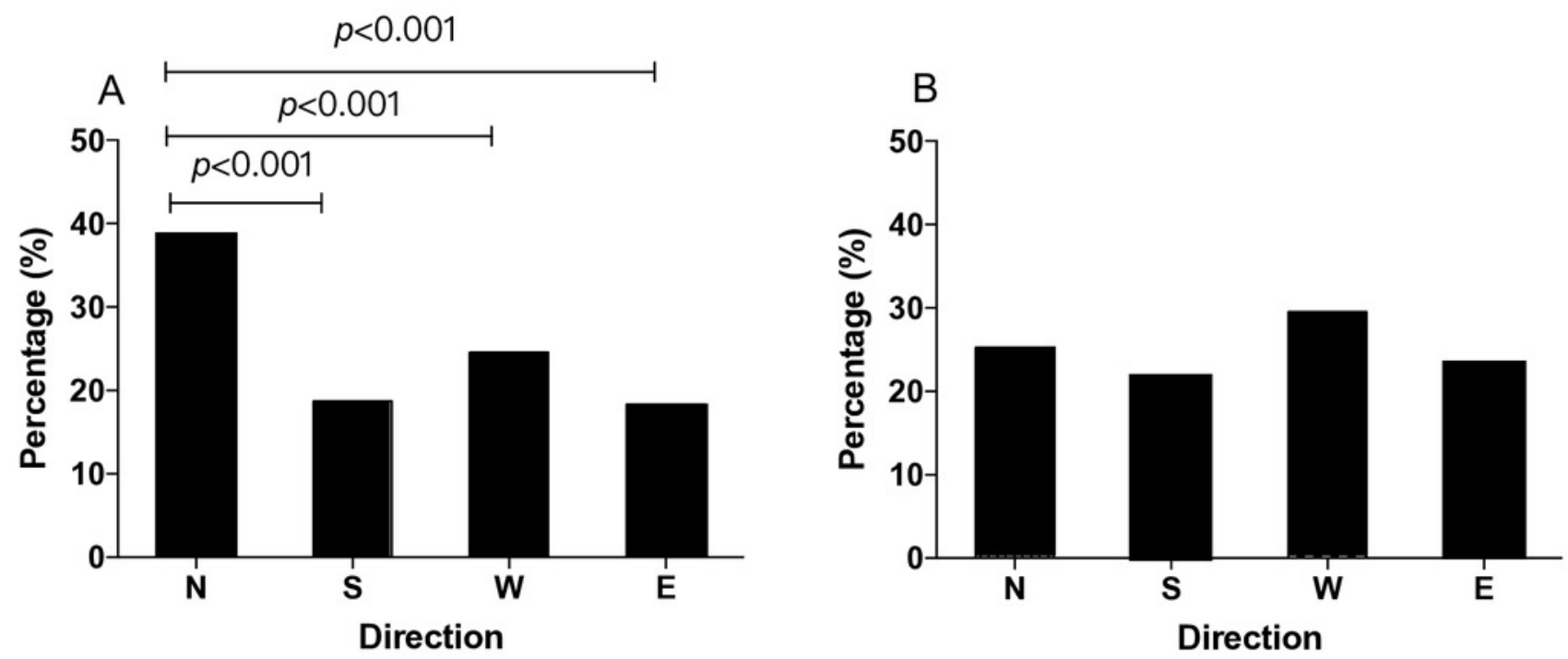
Figure 5

The RNAi efficiency for IscAl within 48h after microinjection.

The relative expression level was quantified with regard to the NI-ISCA1 value injected with dsGFP. The levels are expressed as means \pm SE. Significant differences between $d s G F P$ and $d s N I-I S C A 1$ injection treatments were determined by one-way ANOVA at $p<0.001$. Data points are means of three independent experiments.

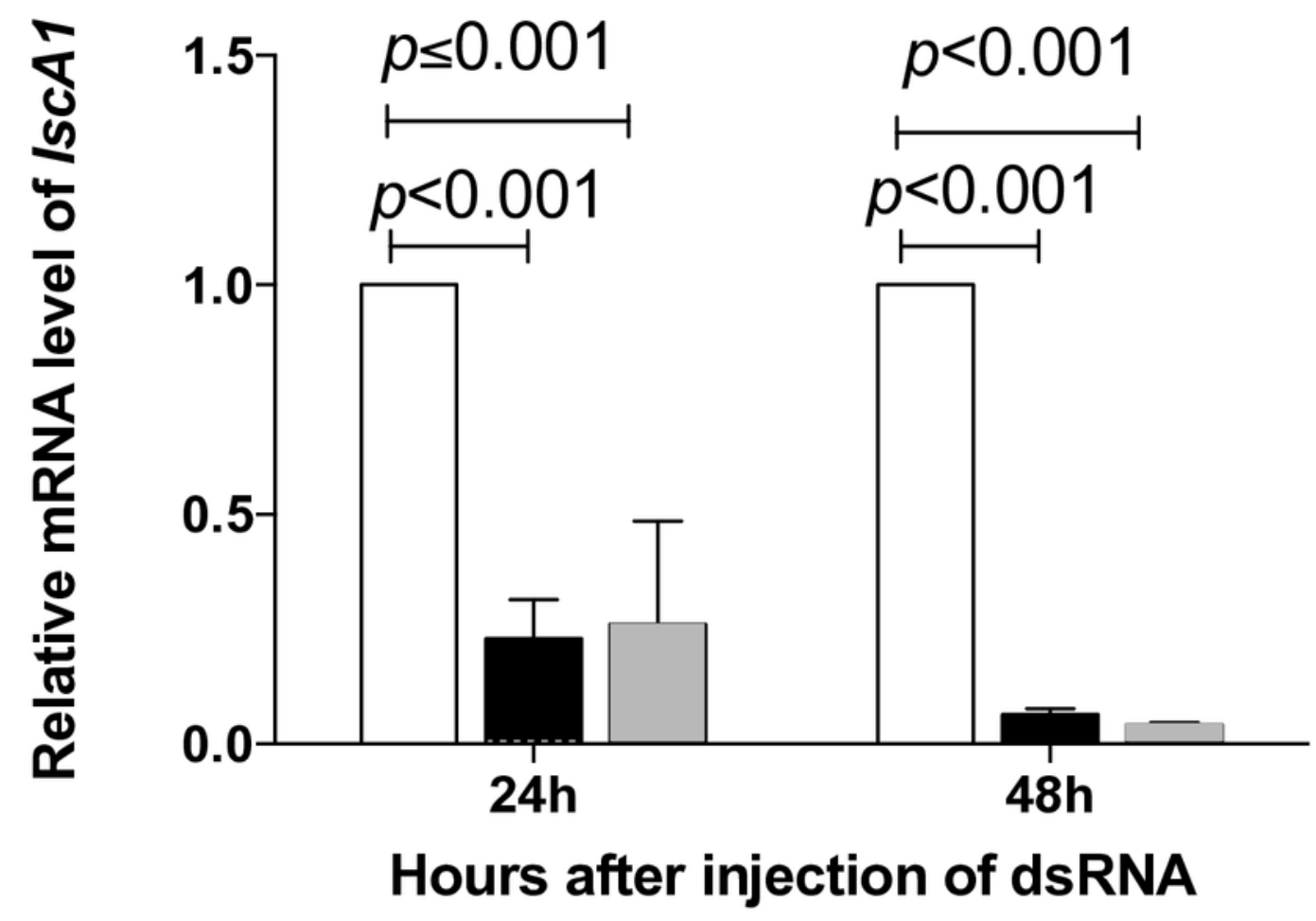

$\square$ dsGFP

- dsRNA1

dsRNA2 


\section{Figure 6}

The distribution of $N$. lugens with the IsCA1 gene knockdown under local GMF $(A)$ and horizontal component reversal of GMF (B) with food source initially located in the north direction.

A total number of insects with $N=151,122,159,92$ for the distribution of IscA1 gene knockdown in the north, south, west and east under GMF and insects with $N=126,101,140$, 74 for the distribution of IscAl gene knockdown in the north, south, west and east under horizontal component reversal of GMF were used for experiments.
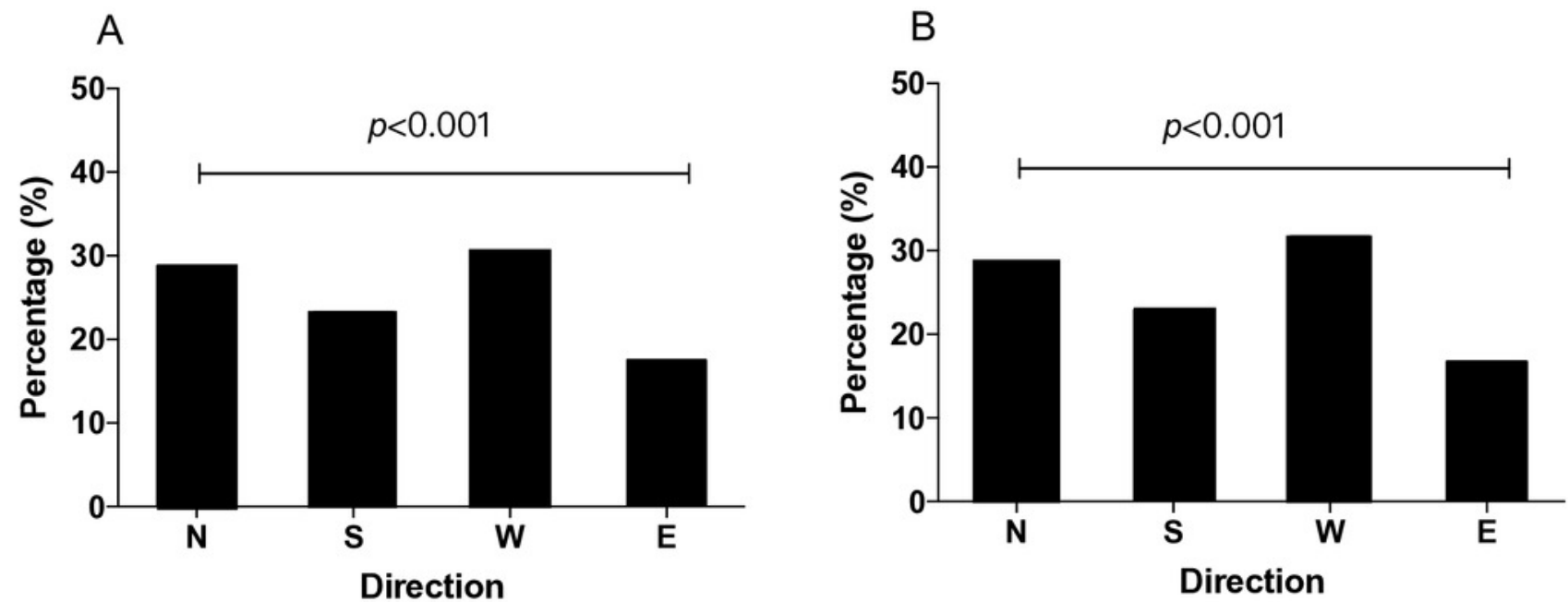